\title{
Ranibizumab pretreatment in diabetic vitrectomy: a pilot randomised controlled trial (the RaDiVit study)
}

O Comyn', L Wickham¹, DG Charteris ${ }^{1}$, PM Sullivan' ${ }^{1}$ E Ezra' 1 Z Gregor ${ }^{1}$, GW Aylward'1 L da Cruz', D Fabinyi ${ }^{1}$, T Peto $^{1,2}$, M Restori ${ }^{1,3}$, W Xing ${ }^{1}$, C Bunce ${ }^{1}$, PG Hykin ${ }^{1}$ and JW Bainbridge ${ }^{1}$

\begin{abstract}
Purpose Our aim was to evaluate the impact of intravitreal ranibizumab pretreatment on the outcome of vitrectomy surgery for advanced proliferative diabetic retinopathy. The objective was to determine the feasibility of a subsequent definitive trial and estimate the effect size and variability of the outcome measure.

Patients and methods We performed a pilot randomised double-masked single-centre clinical trial in 30 participants with tractional retinal detachment associated with proliferative diabetic retinopathy. Seven days prior to vitrectomy surgery, participants were randomly allocated to receive either intravitreal ranibizumab (Lucentis, Novartis Pharmaceuticals UK Ltd, Frimley, UK) or subconjunctival saline (control). The primary outcome was best-corrected visual acuity 12 weeks following surgery.
\end{abstract}

Results At 12 weeks, the mean (SD) visual acuity was 46.7 (25) ETDRS letters in the control group and 52.6 (21) letters in the ranibizumab group. Mean visual acuity improved by 14 (31) letters in the control group and by 24 (27) letters in the ranibizumab group. We found no difference in the progression of tractional retinal detachment prior to surgery, the duration of surgery, or its technical difficulty. Vitreous cavity haemorrhage persisted at 12 weeks in two of the control group but none of the ranibizumab group.

Conclusion Ranibizumab pretreatment may improve the outcome of vitrectomy surgery for advanced proliferative diabetic retinopathy by reducing the extent of postoperative vitreous cavity haemorrhage. However, the effect size appears to be modest; we calculate that a definitive study to establish a minimally important difference of 5.9 letters at a significance level of $\boldsymbol{P}<0.05$ would require 348 subjects in each arm. Eye (2017) 31, 1253-1258; doi:10.1038/eye.2017.75; published online 12 May 2017

\section{Introduction}

Advanced proliferative retinopathy is characterised by fibrovascular proliferation, vitreous haemorrhage, and tractional retinal detachment (Figures 1a and b). This condition is conventionally managed by vitreoretinal surgery, the outcome of which can be limited by recurrent post-operative vitreous cavity haemorrhage. ${ }^{1,2}$

The intraocular administration of therapeutic antivascular endothelial growth factor (VEGF) antibodies is variably used as an adjunct to vitrectomy with the aim of improving the outcome by facilitating safe delamination of fibrovascular membranes and reducing the incidence of post-operative vitreous cavity haemorrhage. ${ }^{3-8}$ However, the value of adjunctive administration of anti-VEGF antibodies has yet to be established with confidence.

Intravitreal ranibizumab pretreatment, 7 days prior to vitrectomy surgery for diabetic tractional retinal detachment, can reduce intraoperative haemorrhage. ${ }^{9}$ The aim of the present study was to evaluate its impact on postoperative outcomes. The objective was to measure the effect on visual acuity and ascertain the number of participants that would be needed to determine such an effect with confidence.

\section{Materials and methods}

We performed a randomised double-masked parallel group pilot study at Moorfields Eye Hospital (NCT01306981). The study conformed
${ }^{1}$ National Institute for Health Research (NIHR) Biomedical Research Centre at Moorfields Eye Hospital and University College London (UCL) Institute of Ophthalmology, London, UK

${ }^{2}$ Reading Centre, Moorfields Eye Hospital, London, UK

${ }^{3}$ Department of Ultrasound, Moorfields Eye Hospital, London, UK

Correspondence: JW Bainbridge, National Institute for Health Research (NIHR) Biomedical Research Centre at Moorfields Eye Hospital and University College London (UCL) Institute of Ophthalmology, 11-43 Bath Street, London EC1V 9EL, UK

Tel: +44 (0)20 7608 4023; Fax: +44 (0)20 76086991. Email: j.bainbridge@ ucl.ac.uk

Received: 1 January 2017 Accepted in revised form: 2 April 2017 Published online: 12 May 2017 
a

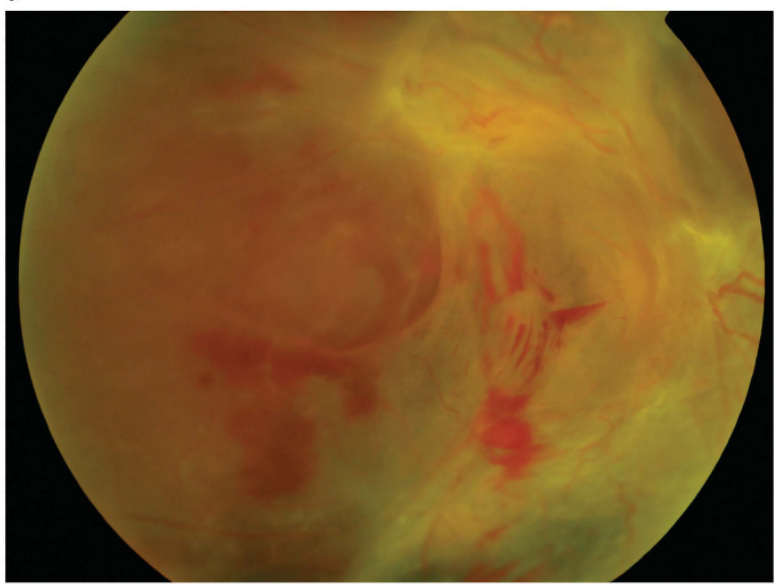

b

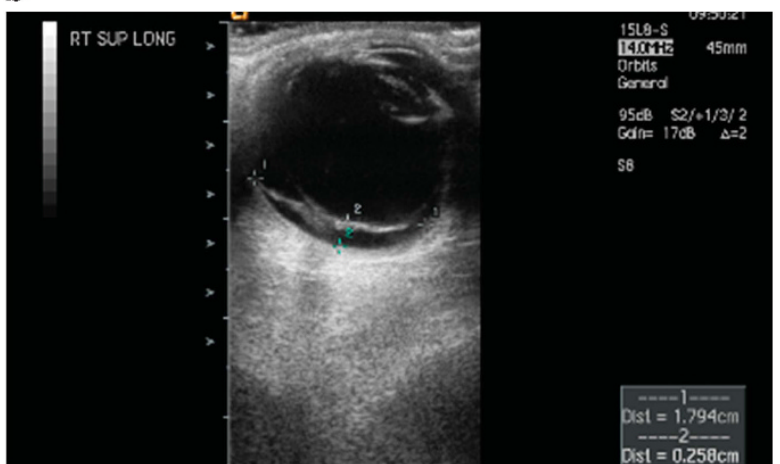

Figure 1 Colour fundus photograph (a) and ultrasound image (b) to show advanced proliferative diabetic retinopathy. The fundus image shows evidence of fibrovascular proliferation, preretinal haemorrhage, and tractional retinal detachment involving the macula, while the ultrasound shows partial posterior vitreous detachment with vitreous attachment to tractional retinal detachment. Calliper placement shows measurement of height and longitudinal base dimension of tractional detachment.

to the Declaration of Helsinki and was approved prospectively by the Central London Research Ethics Committee 1 of the UK National Research Ethics Service. All participants gave their informed consent to participate in the research prior to enrolment.

We included 30 eyes of 30 adult participants having vitrectomy and delamination surgery for advanced proliferative diabetic retinopathy with fibrovascular complexes and/or tractional retinal detachment. Eyes with persistent vitreous haemorrhage could be included because of the use of ultrasonography to evaluate attachment of the retina. We excluded eyes having planned combined cataract and vitrectomy, those with only a single focal point of vitreoretinal attachment apparent on clinical examination or ultrasonography, and those with cataract or uncontrolled glaucoma. We excluded individuals with visual acuity in the contralateral eye of $3 / 60$ or poorer, hypersensitivity to the active substance or excipients, and cerebrovascular, cardiovascular or peripheral vascular disease.

Seven days ( \pm 1 day) prior to vitrectomy surgery, participants were randomly allocated $1: 1$ to receive either intravitreal ranibizumab (Lucentis, $0.5 \mathrm{mg}$ in $0.05 \mathrm{ml}$ solution for injection, Novartis Pharmaceuticals UK Ltd), or subconjunctival saline control (sodium chloride, $0.05 \mathrm{ml}$ of $0.9 \%$ solution for injection) using a $1 \mathrm{ml}$ syringe and 30 gauge needle. Randomisation was performed using random permuted blocks of varying sizes. The allocation sequence was held by the trial statistician, and concealed from the investigator enrolling and assessing participants. An unmasked investigator administered the study agents. Participants in both groups were prepared identically using topical anaesthetic and povidone iodine; topical levofloxacin was administered immediately prior to the injection and four times daily for 4 days. The participants, operating surgeons, and assessing investigators were masked to treatment allocation. Participants were assessed at baseline, immediately prior to surgery, and at 6 and 12 weeks following surgery.

Experienced vitreoretinal surgeons performed 20 gauge pars plana vitrectomy and en bloc delamination of fibrovascular membranes, panretinal endophotocoagulation, with retinopexy and endotamponnade if indicated. We recorded the duration of surgery, the number of back-flush cannula, and endodiathermy applications required to control haemorrhage, retinal breaks, intraoperative bleeding score ( 0 -none; 1 -mild, stopped by bottle elevation; 2 moderate, forming clots or persistent; 3 -severe, covering half of posterior pole), and the anticipated surgical complexity score (Castellarin et al). ${ }^{10}$

To investigate the impact of ranibizumab on the extent of tractional retinal detachment prior to surgery, we examined the study eye prior to administration of the study agent, and 1 week later on the day of surgery by slit-lamp biomicroscopy and ultrasonography (Acuson Sequoia 512 scanner, $14 \mathrm{MHz}$ linear probe; Siemens Medical Solutions USA, Mountain View, CA, USA). To investigate the impact on retinal neovascularisation and ischaemia, we performed fluorescein angiography (unless precluded by vitreous haemorrhage); masked assessors at Moorfields Reading Centre measured the greatest linear dimension and area of the foveal avascular zone, and the grade of perifoveal capillary non-perfusion.

The primary outcome measure was Early Treatment Diabetic Retinopathy Study (ETDRS) best-corrected visual acuity 12 weeks following surgery. Secondary outcome measures included the extent of tractional retinal detachment and macular perfusion at the time of surgery; the technical ease of vitrectomy surgery including duration, instrument usage, and intraoperative 
haemorrhage; and the presence of post-operative vitreous cavity haemorrhage. This was graded using a previously described scale from 0 to 3 (0-no haemorrhage, clear view; $1-$ minor haemorrhage with fundus details visible; 2-moderate haemorrhage with only disc and major vessels visible; 3 -severe haemorrhage with fundus details not visible). ${ }^{11}$

For this pilot trial, we performed no formal sample size calculation, but estimated that 30 subjects would be sufficient to explore the feasibility of a subsequent definitive trial and to enable calculation of its sample size. The Trial Steering Group approved a statistical analysis plan prior to the analysis of data. We compared baseline characteristics of the participants allocated to the two treatment arms. Normality was assessed by inspection of histograms. We calculated summary statistics using STATA statistical software (version 12, StataCorp LP, College Station, TX, USA).

\section{Results}

We included 30 eyes of 30 participants and randomly allocated 15 eyes to each arm of the study, and none were lost to follow-up. All eyes had been managed for proliferative retinopathy by panretinal photocoagulation prior to enrolment. The participants in the two arms were similar (Table 1), though the overall complexity score derived from ultrasound assessment of tractional detachment was slightly higher in the ranibizumab group (Table 2).

At 12 weeks following surgery, the mean (SD) visual acuity was 46.7 (25) ETDRS letters in the control group and 52.6 (21) letters in the ranibizumab group (Figure 2). The mean visual acuity improved by 14 (31) letters in the control group and by 24 (27) letters in the ranibizumab group.

One participant in each group developed new tractional retinal detachment following the study injection; in neither instance did this involve the macula (Table 2). The mean height of TRD increased slightly in control eyes following the study injection. No other change in TRD dimension exceeded the limit of resolution $( \pm 1.6 \mathrm{~mm})$ and the overall ultrasound-derived complexity score was unchanged in both groups.

The median duration of surgery was greater in the ranibizumab group (63 $\mathrm{min}$ ) than the control group (51 min) (Table 3; $P=0.53$ ); intraoperative haemorrhage scores were similar as were the number of endodiathermy and back-flush cannula applications. The median surgeon-defined complexity score based on retinal features present at the start of surgery was slightly higher for the ranibizumab group, but the median overall subjective surgical difficulty score was lower and there were fewer iatrogenic retinal breaks.
Table 1 Participants in a pilot randomised study of ranibizumab pretreatment in diabetic vitrectomy

\begin{tabular}{|c|c|c|}
\hline & Control & Ranibizumab \\
\hline Total number of participants & 15 & 15 \\
\hline Female gender (number of participants) & $9(60 \%)$ & $3(20 \%)$ \\
\hline Age (years); mean (SD) & $48.7(18)$ & $57.1(14)$ \\
\hline \multicolumn{3}{|l|}{ Ethnicity: (number of participants) } \\
\hline White or White British & $8(53 \%)$ & $8(53 \%)$ \\
\hline Black or Black British & $2(13 \%)$ & $2(13 \%)$ \\
\hline Asian or Asian British & $2(13 \%)$ & $3(20 \%)$ \\
\hline Other & $3(20 \%)$ & $1(7 \%)$ \\
\hline Not recorded & 0 & $1(7 \%)$ \\
\hline \multicolumn{3}{|l|}{ Type of diabetes } \\
\hline Type 1 (number of participants) & $6(40 \%)$ & $4(27 \%)$ \\
\hline Type 2 (number of participants) & $9(60 \%)$ & $11(73 \%)$ \\
\hline $\begin{array}{l}\text { Duration of diabetes (years); } \\
\text { median (IQR) }\end{array}$ & $21(15,28)$ & $19(10,23)$ \\
\hline Systolic blood pressure $(\mathrm{mm} \mathrm{Hg})$ & $132(22)$ & $128(22)$ \\
\hline Diastolic blood pressure $(\mathrm{mm} \mathrm{Hg})$ & $75(9)$ & $78(11)$ \\
\hline $\mathrm{HbA}_{1 \mathrm{C}}$ (glycosylated haemoglobin, \%) & $9.3(1.8)(n=13)$ & $8.2(1.1)(n=14)$ \\
\hline \multicolumn{3}{|l|}{ Ocular characteristics } \\
\hline ETDRS letter score & $32.3(19)$ & $28.5(27)$ \\
\hline RAPD present (number) & $3(20 \%)$ & $4(27 \%)$ \\
\hline Rubeosis present (number) & 0 & 0 \\
\hline $\begin{array}{l}\text { Anterior chamber inflammation } \\
\text { grade }(0-4)\end{array}$ & 0 & 0 \\
\hline Intraocular pressure (mm Hg) & $16.5(4)$ & $16.8(3)$ \\
\hline \multicolumn{3}{|l|}{ Lens status } \\
\hline Pseudophakic & $3(20.0 \%)$ & $5(33.3 \%)$ \\
\hline Cataract grade 0 & $4(26.7 \%)$ & $4(26.7 \%)$ \\
\hline 1 & $6(40.0 \%)$ & $5(33.3 \%)$ \\
\hline 2 & $1(6.7 \%)$ & $1(6.7 \%)$ \\
\hline 3 & $1(6.7 \%)$ & 0 \\
\hline 4 & 0 & 0 \\
\hline \multicolumn{3}{|l|}{ Vitreous haemorrhage score } \\
\hline 0 (no haemorrhage) & $3(20.0 \%)$ & $6(40.0 \%)$ \\
\hline 1 (minor haemorrhage) & $8(53.3 \%)$ & $5(33.3 \%)$ \\
\hline $\begin{array}{l}2 \text { (moderate haemorrhage-disc and } \\
\text { large vessels visible) }\end{array}$ & $2(13.3 \%)$ & $1(6.7 \%)$ \\
\hline $\begin{array}{l}3 \text { (severe haemorrhage - no fundus } \\
\text { view) }\end{array}$ & $2(13.3 \%)$ & $3(20.0 \%)$ \\
\hline
\end{tabular}

Abbreviations: ETDRS, Early Treatment Diabetic Retinopathy Study; RAPD, relative afferent pupillary defect.

Data shown as mean (SD) for normally distributed variables.

Any residual vitreous cavity haemorrhage following vitrectomy surgery resolved progressively in both groups. At 6 weeks after surgery, 2 of 15 subjects in the control group, and 1 of 13 subjects in the ranibizumab group who had not received silicone oil had visible vitreous cavity haemorrhage. Although this was moderate in the ranibizumab group, in the control group both had a severe grade of haemorrhage. Moderate or severe residual haemorrhage persisted in two eyes of the control group at 12 weeks following surgery, but had fully resolved in the subject in the ranibizumab group. 
Table 2 Tractional retinal detachment characteristics following study injection in a randomised trial of ranibizumab prior to diabetic vitrectomy

\begin{tabular}{|c|c|c|c|c|}
\hline \multirow[t]{2}{*}{ Time point } & \multicolumn{2}{|c|}{ Control } & \multicolumn{2}{|c|}{ Ranibizumab } \\
\hline & Baseline & 1 week & Baseline & 1 week \\
\hline \multicolumn{5}{|l|}{ TRD characteristics from ultrasound } \\
\hline Number of eyes with TRD & $6(40 \%)$ & $7(47 \%)$ & $10(67 \%)$ & $11(73 \%)$ \\
\hline Number of eyes with macula TRD involving & $5(83 \%)$ & $5(71 \%)$ & $5(50 \%)$ & $5(45 \%)$ \\
\hline \multicolumn{5}{|l|}{ Dimensions of TRD (mean (SD)) } \\
\hline Height (mm) & $2.2(0.5)$ & $2.5(0.6)$ & $2.4(0.9)$ & $2.4(1.0)$ \\
\hline Base $(\mathrm{mm})$ & $11.9(5.0) \times 13.2(4.6)$ & $12.6(5.3) \times 13.5(4.4)$ & $11.9(4.7) \times 11.5(5.3)$ & $12.1(5.0) \times 12.5(6.2)$ \\
\hline $\begin{array}{l}\text { Ultrasound-derived complexity } \\
\text { score }(0-8 \text {; median) }\end{array}$ & 4 & 4 & 5 & 5 \\
\hline \multicolumn{5}{|l|}{ TRD characteristics from colour imaging (baseline only) } \\
\hline Number with gradable images & 9 & - & 8 & - \\
\hline Absent & 1 & & 4 & \\
\hline Hammock & 3 & & 3 & \\
\hline Diffuse central & 3 & & 0 & \\
\hline Tabletop & 2 & & 1 & \\
\hline
\end{tabular}

Abbreviation: TRD, tractional retinal detachment.

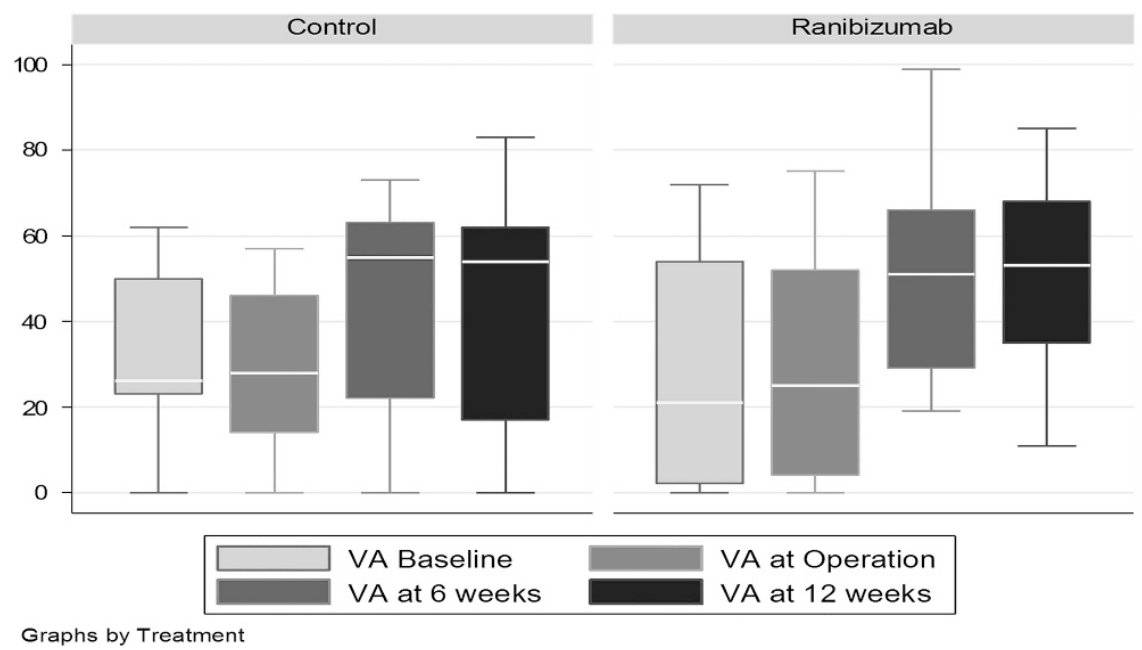

Figure 2 Box plots to show visual acuity (VA) by treatment group, shown as mean \pm SD Early Treatment Diabetic Retinopathy Study letter score.

At baseline, fluorescein angiograms were gradable in only a minority of participants (three of the control group and five of the ranibizumab group) owing to media opacity and/or distortion of foveal anatomy in the majority. All gradable angiograms demonstrated moderate to severe perifoveal capillary non-perfusion. At 12 weeks, the mean (SD) foveal avascular zone greatest linear dimension was $637(236) \mu \mathrm{m}$ in the control group $(n=9)$ and $765(576) \mu \mathrm{m}$ in the ranibizumab group $(n=10)$; the foveal avascular zone area was $0.315(0.147) \mathrm{mm}^{2}$ in the control group and $0.403(0.562) \mathrm{mm}^{2}$ in the ranibizumab group. The median total score for perifoveal capillary non-perfusion was 14 in both groups, indicating scores of 3-4 (moderate to severe) in each of the four quadrants.
Ocular and non-ocular adverse events were in keeping with previously published trials of anti-VEGF agents. The most common adverse event was upper respiratory tract infection that occurred more commonly in the control group. There were no arterial thrombo-embolic events or cases of endophthalmitis. There was one serious adverse event in each group. One participant in the control group was admitted to hospital for management of hypoglycaemia 10 weeks after surgery, and one participant in the ranibizumab group was admitted for management of raised intraocular pressure following vitrectomy surgery, an event judged unlikely to be related to study drug administration. Vitreous cavity haemorrhage was more frequent in the control group than in the ranibizumab group. 
Table 3 Intraoperative parameters in a trial of ranibizumab in diabetic vitrectomy

\begin{tabular}{|c|c|c|c|}
\hline & Control & Ranibizumab & \\
\hline $\begin{array}{l}\text { Surgeon-determined complexity score } \\
0 \text { (simple)-8 (highly complex) (median score) }\end{array}$ & 4 & 5 & \\
\hline $\begin{array}{l}\text { Duration of vitrectomy (min), } \\
\text { median (interquartile range) }\end{array}$ & $51(38-82)$ & $63(42-87)$ & $P=0.53$, Wilcoxon rank-sum test \\
\hline $\begin{array}{l}\text { Intraoperative bleeding score (median score) } \\
0 \text { (none) }-3 \text { (severe) }\end{array}$ & 2 & 2 & \\
\hline Endodiathermy applications (mean) & 1.5 & 1.1 & \\
\hline Back-flush cannula applications (mean) & 2.8 & 2.5 & \\
\hline Number of iatrogenic retinal breaks (total) & 15 & 12 & \\
\hline \multicolumn{4}{|l|}{ Tamponnade agent (number of participants) } \\
\hline None & $3(20 \%)$ & $3(20 \%)$ & \\
\hline Sulphur hexafluoride & $11(73 \%)$ & $9(60 \%)$ & \\
\hline Perfluoropropane & $1(7 \%)$ & $1(7 \%)$ & \\
\hline Silicone oil & $0(0 \%)$ & $2(13 \%)$ & \\
\hline $\begin{array}{l}\text { Overall surgical difficulty score (median score), } \\
\text { scale } 0 \text { (simple)-4 (very difficult) }\end{array}$ & 3 & 2 & \\
\hline
\end{tabular}

\section{Discussion}

The results of this trial confirm significant improvement in mean visual acuity 12 weeks following vitreoretinal surgery for advanced proliferative retinopathy, with or without ranibizumab pretreatment, consistent with previous reports. ${ }^{1}$ Our findings also suggest a modest additional benefit of intravitreal injection of ranibizumab 1 week prior to surgery, with higher mean visual acuity and greater mean improvement in visual acuity at 12 weeks. The difference in acuity in this pilot study is not statistically significant. On the basis of these data, we calculate that 348 subjects in each group (696 in total) would be required to determine a clinically relevant treatment difference of 5.9 letters with $90 \%$ power and $5 \%$ significance, allowing for $5 \%$ loss to follow-up.

Our findings of reduced post-operative vitreous cavity haemorrhage associated with ranibizumab pretreatment in vitrectomy surgery are consistent with a previous report that this also reduces intraoperative haemorrhage during surgery. ${ }^{9}$ Earlier studies have shown conflicting results regarding the utility of bevacizumab in preventing recurrent haemorrhage; for example, Romano et al ${ }^{12}$ found that although pre-operative injection could reduce the number of recurrent haemorrhages, giving the drug as an intraoperative adjunct did not prevent post-operative vitreous cavity haemorrhage. ${ }^{11}$ Data from the 2015 update to the Cochrane review of bevacizumab for the prevention of post-operative vitreous cavity haemorrhage suggest that treatment results in 130 fewer people per 1000 experiencing early post-operative haemorrhage, although there is considerable heterogeneity of methodology in the trials included in this systematic review. ${ }^{8}$

We found that ranibizumab pretreatment was associated with a lower intraoperative bleeding score, greater reduction in retinal neovascularisation, and lower prevalence of persistent vitreous cavity haemorrhage. However, in contrast to previous studies and a metaanalysis of these studies that have reported shorter surgical duration or fewer instrument exchanges following anti-VEGF prior to surgery for proliferative diabetic retinopathy, 4,7 we found that the median surgical complexity score was no lower, the mean duration of vitrectomy was no shorter, and the use of the back-flush cannula and endodiathermy were similar.

Previous studies have highlighted concerns about the development or progression of tractional retinal detachment associated with progressive fibrosis following intravitreal administration of anti-VEGF agents, especially in the absence of prior panretinal photocoagulation. ${ }^{13,14}$ Despite the presence of dense media opacity in many, we were able to determine the impact of ranibizumab pretreatment on the extent of tractional retinal detachment prior to surgery in all participants by the use of ultrasonography. In our study, in which all subjects had been managed previously by panretinal photocoagulation, we identified no effect of ranibizumab injection on extension of retinal detachment after 7 days. We chose this interval between injection and surgery to maximise the possibility of benefit while minimising the risk of harm, and this finding from ultrasound evaluation suggests that administering ranibizumab in the setting of previously treated proliferative retinopathy may be safe in this regard.

We identified significant macular ischaemia in the participants in both groups, but found no evidence of an impact of intervention in either. Although we conclude that its safety profile appears to be favourable, the number of subjects in whom angiography was feasible is insufficient to draw firm conclusions about the impact of ranibizumab injection on the extent of macular ischaemia in this context. 
The strengths of our study are that it was randomised and double-masked with a sham control arm. Since the study included multiple surgeons, all of whom were experienced in vitrectomy surgery for advanced diabetic retinopathy, the findings are broadly generalisable. The ability to draw firm conclusions is limited by the small number of subjects included and the inherent heterogeneity of the condition, in particular, the variability of management of proliferative retinopathy prior to enrolment in the study and difficulty in controlling for variable amounts of prior panretinal photocoagulation. However, the results provide a valuable indication of the substantial size of the trial that would be needed to confirm with confidence the impact of ranibizumab pretreatment in vitrectomy surgery for advanced diabetic retinopathy.

\section{Summary}

\section{What was known before}

- Anti-VEGF agents are in widespread use as adjuncts to vitreoretinal surgery for advanced proliferative retinopathy. Detailed data on surgical outcomes are limited to small uncontrolled studies, although there is now good quality evidence that prior injection of bevacizumab reduces post-operative vitreous cavity haemorrhage. Ranibizumab has been reported to reduce intraoperative haemorrhage.

\section{What this study adds}

- This pilot randomised clinical trial investigated ranibizumab pretreatment before diabetic vitrectomydelamination surgery. The study shows that the likely effect size of ranibizumab on final visual acuity is likely to be small, and with the heterogeneity of outcomes in this condition, a further definite study powered to detect a significant difference is unlikely to be feasible given the subject numbers required. Clinicians will likely therefore continue to use anti-VEGF agents in this condition based on individual experience and preference.

\section{Conflict of interest}

OC has received travel support from Novartis. The remaining authors declare no conflict of interest.

\section{Acknowledgements}

We are grateful to David Yorston and Edward Hughes for constructive advice on design of the trial. This study was supported by an unrestricted research grant from Novartis Pharmaceuticals UK Ltd; the National Institute for Health Research Biomedical Research Centre at Moorfields Eye Hospital National Health Service Foundation Trust and University College London Institute of Ophthalmology; and a multi-user equipment grant from The Wellcome Trust (099173/Z/12/Z).

\section{References}

1 Yorston D, Wickham L, Benson S, Bunce C, Sheard R, Charteris D. Predictive clinical features and outcomes of vitrectomy for proliferative diabetic retinopathy. $\mathrm{Br} \mathrm{J}$ Ophthalmol 2008; 92: 365-368.

2 Schiff WM, Barile GR, Hwang JC, Tseng JJ, Cekiç O, Del Priore LV et al. Diabetic vitrectomy: influence of lens status upon anatomic and visual outcomes. Ophthalmology 2007; 114: 544-550.

3 Chen E, Park C. Use of intravitreal bevacizumab as a preoperative adjunct for tractional retinal detachment repair in severe proliferative diabetic retinopathy. Retina 2006; 26 : 699-700.

4 Rizzo S, Genovesi-Ebert F, Di Bartolo E, Vento A, Miniaci S, Williams G. Injection of intravitreal bevacizumab (Avastin) as a preoperative adjunct before vitrectomy surgery in the treatment of severe proliferative diabetic retinopathy (PDR). Graefes Arch Clin Exp Ophthalmol 2008; 246: 837-842.

5 Ahmadieh H, Shoeibi N, Entezari M, Monshizadeh R. Intravitreal bevacizumab for prevention of early postvitrectomy hemorrhage in diabetic patients: a randomized clinical trial. Ophthalmology 2009; 116: 1943-1948.

6 di Lauro R, De Ruggiero P, di Lauro R, di Lauro MT, Romano MR. Intravitreal bevacizumab for surgical treatment of severe proliferative diabetic retinopathy. Graefes Arch Clin Exp Ophthalmol 2010; 248: 785-791.

7 Zhao LQ, Zhu H, Zhao PQ, Hu YQ. A systematic review and meta-analysis of clinical outcomes of vitrectomy with or without intravitreal bevacizumab pretreatment for severe diabetic retinopathy. Br J Ophthalmol 2011; 95: 1216-1222.

8 Smith JM, Steel DH. Anti-vascular endothelial growth factor for prevention of postoperative vitreous cavity haemorrhage after vitrectomy for proliferative diabetic retinopathy. Cochrane Database Syst Rev 2015; (8): CD008214.

9 Ribeiro JA, Messias A, de Almeida FP, Costa RA, Scott IU, de Figueiredo-Pontes LL et al. The effect of intravitreal ranibizumab on intraoperative bleeding during pars plana vitrectomy for diabetic traction retinal detachment. $\mathrm{Br} J$ Ophthalmol 2011; 95: 1337-1339.

10 Castellarin A, Grigorian R, Bhagat N, Del Priore L, Zarbin MA. Vitrectomy with silicone oil infusion in severe diabetic retinopathy. Br J Ophthalmol 2003; 87: 318-321.

11 Romano M, Gibran S, Marticorena J, Wong D, Heimann H. Can an intraoperative bevacizumab injection prevent recurrent postvitrectomy diabetic vitreous hemorrhage? Eur J Ophthalmol 2009; 19: 618-621.

12 Romano MR, Gibran SK, Marticorena J, Wong D, Heimann H. Can a preoperative bevacizumab injection prevent recurrent postvitrectomy diabetic vitreous haemorrhage? Eye 2009; 23: 1698-1701.

13 Arevalo JF, Maia M, Flynn HW, Saravia M, Avery RL, Wu L et al. Tractional retinal detachment following intravitreal bevacizumab (Avastin) in patients with severe proliferative diabetic retinopathy. Br J Ophthalmol 2007; 92: 213-216.

14 Van Geest RJ, Lesnik-Oberstein SY, Tan HS, Mura M, Goldschmeding R, Van Noorden CJ et al. A shift in the balance of vascular endothelial growth factor and connective tissue growth factor by bevacizumab causes the angiofibrotic switch in proliferative diabetic retinopathy. $\mathrm{Br} J$ Ophthalmol 2012; 96: 587-590. 